\title{
Microcistina en plantas de tratamiento de agua para consumo humano en un ambiente tropical: el Área Metropolitana de Costa Rica
}

\author{
Adrián Avendaño Lopez ${ }^{1} \&$ Carolina Arguedas Villa ${ }^{2}$ \\ 1 Centro de Investigación en Enfermedades Tropicales (CIET), Departamento de Parasitología, Facultad de \\ Microbiología, Universidad de Costa Rica, San José, Costa Rica; aeavenda@cariari.ucr.ac.cr \\ 2 Laboratorio de Análisis Ambiental. Escuela de Ciencias Ambientales, Facultad de Ciencias de la Tierra y el Mar, \\ Universidad Nacional, Heredia, Costa Rica; cargue@una.ac.cr
}

Recibido 25-IV-2005. Corregido 23-I-2006. Aceptado 23-V-2006.

\begin{abstract}
Microcystin in plants that treat water for human consumption in a tropical environment: the Metropolitan Area of Costa Rica. We measured microcystin levels in water of the Metropolitan Area of Costa Rica by competitive inhibition ELISA and we quantified total coliforms, fecal coliforms, Escherichia coli (by a Most Probable Number method) and aerobic count. We wanted to identify any cyanotoxin correlation with these parameters, as a public health risk. We sampled in the rainy season of 2003 (April-October) and in the dry season of 2004 (February-March) (30 samples/season). We sampled pre-treated, semi-treated and treated water. Microcystin levels $<0.5 \mathrm{ppb}$ were found in the rainy season (and $>0.5 \mathrm{ppb}$ in the dry season). Dry season levels exceeded World Health Organization limits $(1.0 \mathrm{ppb})$. Cyanotoxins occurred in the Tres Ríos plant. We did not find a correlation between these microbiologic parameters of water quality and microcystin levels in water. Rev. Biol. Trop. 54 (3): 711-716. Epub 2006 Sept. 29.
\end{abstract}

Key words: Microcystin, cyanotoxin, treated water, fecal coliforms, Metropolitan Area Costa Rica.

Las cianobacterias son agentes procariotas, autótrofos, fotosintéticos cuyo desarrollo se presenta en forma de células aisladas, colonias, o tricomas. Su origen se estima hace más de 3 millones de años y se asocia con largos procesos de evolución donde han colaborado en la producción de materia orgánica y la deposición de oxígeno en la atmósfera (Anónimo 2002c). Las cianobacterias proliferan formando espumas superficiales sobre el agua, las cuales tienen una alta densidad de población de diferentes especies de cianobacterias (Carmichael 1994).

Las proliferaciones de cianobacterias se desarrollan en aguas eutróficas, ecosistemas artificialmente enriquecidos por las actividades humanas, con reducción del oxígeno disuelto y muerte de la fauna y flora natural que conforman el ecosistema. Algunas cianobacterias tienen la capacidad de producir cianotoxinas, metabolitos secundarios cuya producción es máxima en condiciones óptimas de crecimiento, por lo tanto la producción de cianotoxinas es directamente proporcional al aumento de biomasa. Las cianotoxinas se han descrito químicamente como péptidos cíclicos con una cadena lateral de aminoácidos (ADDA: ácido 3 amino-9-metoxi-2,6,8-trimetil-10-fenildeca4,6-dienoico), alcaloides y fosfatos orgánicos. Entre las especies productoras de toxinas se han informado: Microcystis sp., Nodularia sp., Anabaena sp., Oscillatoria sp. y Phaktotrix sp. (Anónimo 2002b).

Las cianotoxinas son capaces de producir cuadros hepatotóxicos y neurotóxicos en vertebrados superiores. Las hepatotoxinas son las que se describen con mayor frecuencia en los casos de intoxicación por toxinas de 
cianobacterias; su mecanismo de acción es lento y se caracteriza por generar hemorragias intra hepáticas y choques hipovolémicos (Anónimo 2002b).

Las microcistinas son las hepatotoxinas informadas con mayor frecuencia. Generan un cuadro de necrosis hepática en pocas $\mathrm{h}$ o días cuando es administrada en una dosis letal, por el contrario en dosis bajas no letales se afirma que el efecto tóxico de la microcistina es crónico y acumulativo (Carmichael 1994).

En Latinoamérica se informan casos clínicos asociados con el consumo de agua con cianotoxinas; en Brasil se ha informado la muerte de pacientes por ingesta de cianotoxinas. Con base en los antecedentes, la Organización Mundial de la Salud (OMS) y la Organización Panamericana de la Salud (OPS), han implementado planes de control y monitoreo de cianotoxinas, asociado al control de la calidad del agua de consumo humano (Agujaro e Isaac 2003).

En Costa Rica se carece de estudios relacionados con concentraciones de cianotoxinas en aguas de consumo humano, descripción de hepatotoxinas o microcistinas ni ninguna estrategia de control, hasta la fecha. Se han descrito cianobacterias potencialmente tóxicas presentes en floramientos de plantas de tratamiento de agua (Peinador 1992) y por otro lado, se les ha dado un papel como indicadores de contaminación orgánica (Peinador 1999)

En el presente trabajo se da a conocer el primer informe de la presencia de microcistinas en una de las plantas de tratamiento estudiadas en Costa Rica, por medio de una técnica de ensayo inmunoenzimático (ELISA).

\section{MATERIALES Y MÉTODOS}

Se realizaron dos muestreos en cada una de las plantas de tratamiento evaluadas. El primer muestreo se hizo durante la época lluviosa (abril-octubre 2003), y el segundo se llevó a cabo durante la época seca (febreromarzo 2004). Las muestras recolectadas se utilizaron para la detección inmunoenzimática de microcistina y para el análisis microbiológico de aguas. Se tomaron en dos puntos: a la entrada de la planta (muestras de agua pretratada) y a la salida de la planta (muestras de agua tratada). Las muestras de la época lluviosa se colectaron en frascos estériles de vidrio con tapa esmerilada y las de la época seca en bolsas estériles de plástico especiales para recolección de agua, esto con el fin de comprobar el efecto de estos materiales en la concentración de microcistina en una muestra de agua, según se ha informado en la literatura (Metcalf et al. 2000). Durante el segundo muestreo en algunas plantas se recogió una tercera muestra de agua semitratada, correspondiente a la parte superficial de los sedimentadores de las plantas de tratamiento. Todas las muestras fueron congeladas a $-20{ }^{\circ} \mathrm{C}$ previamente a su análisis inmunoenzimático. El análisis de coliformes fecales y termotolerantes se realizó dentro de las $24 \mathrm{~h}$ posteriores a la toma de la muestra.

Para la determinación de microcistina se utilizó un ensayo inmunoenzimático por inhibición competitiva, perteneciente a la casa comercial Envirologix Inc (Portland, ME 04103 USA), con capacidad para detectar cuatro tipos de microcistina y uno de nodularina. Para realizar el ELISA se tomaron $250 \mu \mathrm{l}$ de diluente de ensayo y $100 \mu \mathrm{l}$ de cada muestra (concentrada) por analizar, además de los calibradores con concentraciones de $0.5 \mathrm{ppb}$ (partes por billón) y $3.0 \mathrm{ppb}$. Posteriormente se depositaron en los tubos provistos por la casa comercial, como cubeta de reacción, los cuales estaban recubiertos con el anticuerpo que participa en la detección. Se llevó a cabo una primera incubación de 5 min a temperatura ambiente, luego de ésta se agregaron $250 \mu \mathrm{l}$ de conjugado. Inmediatamente se dio inicio a una incubación de 20 min a temperatura ambiente después de la cual se realizaron tres lavados con agua destilada para agregar en seguida $500 \mu 1$ de sustrato. Finalmente, pasados $10 \mathrm{~min}$, una vez que se agregó el sustrato a cada tubo se adicionaron $700 \mu \mathrm{l}$ de una solución para detener la reacción con el fin de parar el desarrollo de color. La lectura de calibradores y cada muestra se realizó en un espectrofotómetro Shimadzu UV-160, 
a una longitud de onda de $450 \mathrm{~nm}$. Con base en los resultados obtenidos se ejecutaron diluciones del calibrador de $3.0 \mathrm{ppb}$ para hacer una curva de calibración con concentraciones de $0.5,1.0,1.5$ y $3.0 \mathrm{ppb}$.

Para el análisis microbiológico de aguas se llevó a cabo un análisis de coliformes termotolerantes y totales, mediante la técnica de Número Más Probable (NMP) y un recuento total aerobio psicrófilo, ambos procedimientos se efectuaron siguiendo los protocolos establecidos por la American Public Health Association (Anónimo 1992).

\section{RESULTADOS}

No se detectó presencia de cianotoxinas durante la estación lluviosa del 2003 en ninguna de las plantas evaluadas, por el contrario se descubrió microcistina durante la estación seca del 2004.

En la estación lluviosa del 2003 se procesaron un total de 30 muestras provenientes de las plantas de tratamiento citadas en el Cuadro 1, con excepción de la planta de Coronado. En todas las muestras analizadas la concentración de microcistina fue menor a las $0.5 \mathrm{ppb}$.
Referente al análisis microbiológico se señala que el recuento total de la Planta de Tres Ríos indica que el rendimiento de la planta es óptimo, según parámetros microbiológicos (Anónimo 1992).

Durante la estación seca del 2004 se procesaron un total de 30 muestras provenientes de las plantas del Cuadro 1, con excepción de la planta de Alajuelita (Cuadro 2). Dos de las 30 muestras $(7 \%)$ presentaron concentraciones superiores a $0.5 \mathrm{ppb}$ de microcistina o nodularina, esas dos muestras positivas fueron de agua pretratada $(2 / 15=13 \%)$. De las plantas de tratamiento, las dos muestras con concentraciones mayores a las $0.5 \mathrm{ppb}$ fueron obtenidas de la Planta Alta de Tres Ríos $(2 / 6=33 \%)$.

\section{DISCUSIÓN}

Los niveles de microcistina detectados en Tres Ríos durante la estación seca del año 2004, superan el valor máximo aceptado como consumo diario, oral, de la toxina estipulado por la Organización Mundial de la Salud, el cual corresponde a $1.0 \mathrm{ppb}$ (Agujaro e Isaac 2003). Estos niveles superiores a la norma, fueron hallados en agua pretratada de la planta.

CUADRO 1

Niveles de microcistina en aguas de consumo humano del Área Metropolitana, Costa Rica, abril-octubre del 2003 (detección por ELISA)

TABLE 1

Levels of microcystin in waters for human consumption from the Metropolitan Area of Costa Rica, April-October 2003 (detection by ELISA)

\begin{tabular}{|c|c|c|c|c|c|c|}
\hline \multirow[t]{2}{*}{ Planta } & \multicolumn{2}{|c|}{$\begin{array}{l}\text { Muestreo } 1 \\
\text { ppb }\end{array}$} & \multicolumn{2}{|c|}{$\begin{array}{c}\text { Muestreo } 2 \\
\text { ppb }\end{array}$} & \multicolumn{2}{|c|}{$\begin{array}{c}\text { Muestreo } 3 \\
\text { ppb }\end{array}$} \\
\hline & Cruda & Tratada & Cruda & Tratada & Cruda & Tratada \\
\hline Tres Ríos ${ }^{1}$ & $<0.5$ & $<0.5$ & $<0.5$ & $<0.5$ & $<0.5$ & $<0.5$ \\
\hline Guadalupe $^{2}$ & $<0.5$ & $<0.5$ & $<0.5$ & $<0.5$ & $<0.5$ & $<0.5$ \\
\hline Los Sitios $^{3}$ & $<0.5$ & $<0.5$ & $<0.5$ & $<0.5$ & $<0.5$ & $<0.5$ \\
\hline Alajuelita $^{4}$ & $<0.5$ & $<0.5$ & $<0.5$ & $<0.5$ & $<0.5$ & $<0.5$ \\
\hline SJDD $^{5}$ & $<0.5$ & $<0.5$ & $<0.5$ & $<0.5$ & $<0.5$ & $<0.5$ \\
\hline
\end{tabular}

${ }^{1}\left(9^{\circ} 56^{\prime} 01^{\prime \prime} \mathrm{N} \quad 83^{\circ} 58^{\prime} 10^{\prime \prime} \mathrm{W}\right) ; \quad{ }^{2}\left(9^{\circ} 59^{\prime} 3^{\prime \prime} \mathrm{N} 84^{\circ} 02^{\prime} 18^{\prime \prime} \mathrm{W}\right) ; \quad{ }^{3}\left(9^{\circ} 57^{\prime} 57^{\prime \prime} \mathrm{N} 83^{\circ} 59^{\prime} 51^{\prime \prime} \mathrm{W}\right) ; \quad{ }^{4}\left(9^{\circ} 53^{\prime} 16^{\prime \prime} \mathrm{N} 84^{\circ} 06^{\prime} 35^{\prime \prime} \mathrm{W}\right)$; $5\left(9^{\circ} 52^{\prime} 30^{\prime \prime} \mathrm{N} 84^{\circ} 05^{\prime} 37^{\prime \prime} \mathrm{W}\right)$ 
CUADRO 2

Niveles de microcistina en aguas de consumo humano del Área Metropolitana de Costa Rica, febrero-marzo, 2004 (detección por ELISA)

TABLE 2

Levels of microcystin in waters for human consumption from the Metropolitan Area of Costa Rica, February-March, 2004 (detection by ELISA)

\begin{tabular}{|c|c|c|c|c|c|c|}
\hline \multirow{2}{*}{ Planta } & \multicolumn{2}{|c|}{$\begin{array}{c}\text { Muestreo } 1 \\
\text { ppb }\end{array}$} & \multicolumn{2}{|c|}{$\begin{array}{c}\text { Muestreo } 2 \\
\text { ppb }\end{array}$} & \multicolumn{2}{|c|}{$\begin{array}{c}\text { Muestreo } 3 \\
\text { ppb }\end{array}$} \\
\hline & Cruda & Tratada & Cruda & Tratada & Cruda & Tratada \\
\hline Tres Ríos & $<0.5$ & $<0.5$ & $>3.0$ & $<0.5$ & $0.5-3.0$ & $<0.5$ \\
\hline Guadalupe & $<0.5$ & $<0.5$ & $<0.5$ & $<0.5$ & $<0.5$ & $<0.5$ \\
\hline Los Sitios & $<0.5$ & $<0.5$ & $<0.5$ & $<0.5$ & $<0.5$ & $<0.5$ \\
\hline Coronado $^{6}$ & $<0.5$ & $<0.5$ & $<0.5$ & $<0.5$ & $<0.5$ & $<0.5$ \\
\hline SJDD & $<0.5$ & $<0.5$ & $<0.5$ & $<0.5$ & $<0.5$ & $<0.5$ \\
\hline
\end{tabular}

Las plantas de tratamiento de agua del Área Metropolitana son alimentadas por ríos con agua en movimiento, principalmente el río Virilla. En contraparte, Planta Alta de Tres Ríos se nutre de agua proveniente del río Tiribí y del Embalse El Llano. La microcistina detectada en la planta puede formarse en el embalse, donde las cianobacterias proliferan con facilidad y no propiamente en la planta de tratamiento, donde el agua puede estar en continuo movimiento; sin embargo, las espumas superficiales son capaces de desarrollarse en los tanques de las plantas de tratamiento. La aparición de las cianotoxinas durante la estación seca se asocia con el aumento de la biomasa de cianobacterias en las espumas superficiales de aguas almacenadas (Bittencourt et al. 2001). La formación de las espumas se asocia con el carácter eutrófico y el movimiento de las aguas. En las plantas de tratamiento existen diferencias en la velocidad de movimiento del agua en los distintos contenedores con relación a su función (Anónimo 2002a) y con el volumen de las precipitaciones. Asimismo, las cianobacterias, como organismos fotosintéticos, también dependen de la cantidad de luz solar que reciben durante el día para favorecer su proliferación, otros parámetros como $\mathrm{pH}$, concentración de fósforo, nitrógeno, dióxido de carbono, temperatura entre

otros también afectan la presencia de estos organismos en el agua (Peinador 1994).

Al comparar el nivel de microcistina en el agua pretratada de la planta de Tres Ríos con el nivel obtenido en el agua tratada, se observa una disminución considerable en la concentración de microcistina en la muestra de agua tratada. En esta última muestra, la disminución en el nivel de microcistina se podría explicar por la acción degradativa o inhibitoria de agentes químicos en el agua tratada que han sido agregados para el control de otros microorganismos, y que tienen efecto comprobado sobre la microcistina (Jia et al. 2003, Kull et al. 2004).

Por otro lado, el agua que ingresa a la planta no se mezcla en los tanques de mantenimiento sino que lo hace en los filtros de tratamiento. Por lo tanto, no es posible determinar si este tratamiento fue capaz de afectar la concentración de microcistina, o si en realidad lo que se tiene es un efecto de dilución al mezclarse el agua en los filtros y generar valores por debajo de las $0.5 \mathrm{ppb}$ y los respectivos falsos negativos.

El ámbito de detección de microcistina y nodularina por parte de la técnica entre las $0.5 \mathrm{ppb}$ y $3.0 \mathrm{ppb}$, diseñada con base en el parámetro de la OMS de $1.0 \mathrm{ppb}$, pero que no contempla concentraciones por debajo de las 
$0.5 \mathrm{ppb}$ que también pueden ser detectadas por ensayo inmunoenzimático con un ámbito de detección entre 0.16 y $1.60 \mathrm{ppb}$ (Hee et al. 2001, Magalhaes et al. 2003) Estas concentraciones por debajo de $0.5 \mathrm{ppb}$ se asocian con la capacidad hepatotóxica de cianotoxinas en dosis subletales de muy baja concentración (Aguayo y Muñoz 2001).

Otra alternativa para explicar las diferencias de concentración, reside en el envase de recolección de la muestra y el procesamiento al cual es sometido. Se ha descrito que la concentración de microcistina y nodularina se ve afectada cuando las muestras son recolectadas en envases plásticos en comparación con su recolección en envases de vidrio (Metcalf et al. 2000). Las muestras tomadas durante la estación seca fueron recolectadas en bolsas plásticas, lo que implicaría incluso disminuciones en las concentraciones de las muestras de agua tratada, así en este caso se obtuvieron falsos negativos.

Para demostrar la verdadera ausencia de cianotoxinas debe demostrase la ausencia de cianobacterias mediante técnicas de aislamiento a partir de muestras de las espumas superficiales. No obstante, se ha informado de la presencia de cianotoxinas en ausencia de espumas superficiales y cianobacterias, así como se ha descrito la presencia de cianobacterias no productoras de toxinas (Carmichael 1994), por lo que la presencia o ausencia de un aislamiento no es un elemento que permita descartar la ausencia de toxinas en el agua.

Finalmente, no se encontró ningún tipo de asociación entre los parámetros evaluados para el análisis de contaminación fecal y la presencia de microcistina en las plantas de tratamiento. Las condiciones ambientales que favorecen el crecimiento de coliformes fecales o el crecimiento de cianobacterias, son completamente diferentes. La eutroficación de medios acuáticos implica variaciones importantes en las concentraciones de $\mathrm{CO}_{2}$, fósforo y nitrógeno; variaciones que no son indispensables para el crecimiento de coliformes fecales. Eventualmente un cambio en el $\mathrm{pH}$ del medio donde se detectan cianotoxinas puede generar alguna interferencia en el crecimiento de los coliformes fecales (Anónimo 1992, Branco 1984).

La presencia de microcistinas en el agua de consumo humano representa un problema de salud pública. La detección de microcistina en plantas de tratamiento del Área Metropolitana demuestra la importancia de realizar otras investigaciones y establecer normativas para el país, siguiendo patrones establecidos por organizaciones mundiales que impulsan el control de la toxina. En el nivel de la investigación, es prioritario determinar las especies que conforman las poblaciones de cianobacterias en las plantas de tratamiento y establecer la presencia de especies tóxicas. Por otro lado, el análisis de la acción de agentes químicos como el $\mathrm{CO}_{2}, \mathrm{P}$ y $\mathrm{N}$ y otros microorganismos como bacterias aerobias Gram negativas puede brindar información sobre la capacidad de estos agentes sobre las cianobacterias y sus toxinas (Ishii et al. 2004). Por último se deben plantear, evaluar e implementar estrategias para el control de los niveles de cianotoxinas en el agua de consumo humano. Esto constituye el primer informe de presencia de cianotoxinas en plantas de tratamiento de agua para consumo humano en Costa Rica.

\section{AGRADECIMIENTOS}

Agradecemos al personal de plantas y al de Laboratorio Nacional de Aguas de Acueductos y Alcantarillados, especialmente a Carmen Valiente por su apoyo durante todo el proyecto, a la Facultad de Microbiología, específicamente al Laboratorio de Análisis Clínicos y el Laboratorio de Microbiología de Aguas, a José María Gutiérrez G, por sus sugerencias para mejorar el manuscrito.

\section{RESUMEN}

Se midió la presencia de microcistina en el Área Metropolitana de Costa Rica por la técnica de ELISA de inhibición competitiva y se cuantificó coliformes totales, coliformes termotolerantes, Escherichia coli (por medio de la técnica Numero Más Probable) y recuento total 
aerobio. Se realizaron dos etapas de muestreo, una durante la estación lluviosa del 2003 (abril-octubre) y otra durante la estación seca del 2004 (febrero-marzo), cada una con 30 muestras de agua. Se muestreó agua pretratada, semitratada y tratada. Se determinaron niveles de microcistina $<0.5$ ppb durante la estación lluviosa del 2003, mientras que durante la estación seca, se detectaron concentraciones de microcistina $>0.5 \mathrm{ppb}$. Se informó la presencia de cianotoxinas en la Planta de Tratamiento de Tres Ríos. No se establece correlación entre los parámetros microbiológicos de calidad del agua y las concentraciones de microcistina en el agua. Los estudios deberían considerar la diversidad y toxicidad de cianobacterias en estas plantas, los efectos del tratamiento, y presencia de otros microorganismos y sustancias (dióxido de carbono, fósforo, Nitrógeno), sobre la presencia, estructura y efecto de estas toxinas.

Palabras clave: Microcistina, cianotoxina, agua tratada, coliformes fecales, Área Metropolitana de Costa Rica.

\section{REFERENCIAS}

Agujaro, L. \& R. Isaac. 2003. Floraçoes de cianobacterias potencialmente toxicas nas bacias dos rios Piracicaba, Capivari e Jundíai.-Estado de São Paulo- avaliaçao dos mananciais en relaçao a eutrofiçazão. In 22th Congresso Brasileiro de Engenhaira Sanitária e Ambiental. Asociación Brasileira Engenhaira Sanitaria e Ambiental, São Paulo, Brasil.

Aguayo, R. \& M. Muñoz. 2001. Detección de cianobacterias y sus toxinas. Revisión. Rev. Toxico. 18: 65-71.

Anónimo. 2002a. Instructivo de plantas de tratamiento de filtración rápida. Instituto Nacional de Acueductos y Alcantarillados, San José, Costa Rica. 17-28.

Bittencourt, M., M. Cabral \& J. Yunes. 2001. Cianobacterias tóxicas. Rev. Biotec. 23: 44-47.

Branco, M. 1984. Limnología Sanitaria. Estudio de la polución de Aguas Continentales. Secretaría General de la Organización de Estados Americanos, Programa Regional de Desarrollo Científico y Tecnológico; p. 7-63.

Carmichael, W.W. 1994. The toxins of cianobacteria. Sci. Amer. 270: 78-86.

Hee, O., L. Seok, K. Jee, K. Hee \& Y. Byung. 2001. Seasonal Variation and indirect monitoring of Microcystin concentrations in Daechung reservoir, Korea. Appl. Envirom. Microbiol. 67: 1484-1489.

Ishii, H., M. Nishijima \& T. Abe. 2004. Characterization of degradation process of cyanobacterial hepatotoxins by a gramnegative aerobic bacterium. Wat. Res 38 : 2667-2676.

Jia, R., X. Zhang, W. Zhang, G. Zhang \& Z. Wang. 2003. Fluctuation of microcystins in water plant. J. Envirom. Sci. Health. 38: 2867-2875.

Kull, T., P. Backlund, K. Karlsson \& J. Merilouto. 2004. Oxidation of cyanobacterial hepatotoxin MicrocystinLR by Chlorine Dioxide: reaction kinetics characterization, and toxicity of reaction products. Envirom. Sci. Techno. 38: 6025-6031.

Magalhaes, V., M. Marinho, P. Domingos, A. Oliveira, S. Costa, L. Azevedo \& S. Azevedo. 2003. Microcystins (cyanobacteria hepatotoxins) bioaccumulation in fish and crustaceans from Sepetiba Bay (Brazil, RJ). Toxicon 42: 289-245.

Metcalf, J., P. Hyenstrand, K. Beattie \& G. Codd. 2000. Effects of physicochemical variables and cyanobacterial extracts on the immunoassay of microcystin-LR by two ELISA kits. J. Appl. Microbiol. 89: 532-538.

Peinador, M. 1992. Cianobacterias potencialmente tóxicas en plantas de tratamiento de aguas en Costa Rica. Rev. Biol. Trop. 42: 5-8.

Peinador, M. 1999. Las cianobacterias como indicadores de contaminación orgánica. Rev. Biol. Trop. 47: 381-391.

\section{REFERENCIAS DE INTERNET}

Anónimo. 1992. Standard methods for the examination of Water and Wastewater. American Public Health Association (APHA). (Consultado: 27 de junio, 2004, http://www.apha.org)

Anónimo. 2002b. Algas y cianobacterias en aguas dulces. (Consultado: 10 de enero, 2003, http://cruzroja.org)

Anónimo. 2002c. Floraciones nocivas de cianobacterias. Unidad de pesquisa en cianobacterias. Fundaçao Universidade Federal de Río Grande. (Consultado: 10 de enero, 2003, http://www.cianobacterias.furg.br) 